\title{
Pseudo-fractional ARMA modelling using a double Levinson recursion
}

\author{
M.D. Ortigueira and A.J. Serralheiro
}

\begin{abstract}
The modelling of fractional linear systems through ARMA models is addressed. To perform this study, a new recursive algorithm for impulse response ARMA modelling is presented. This is a general algorithm that allows the recursive construction of ARMA models from the impulse response sequence. This algorithm does not need an exact order specification, as it gives some insights into the correct orders. It is applied to modelling fractional linear systems described by fractional powers of the backward difference and the bilinear transformations. The analysis of the results leads to propose suitable models for those systems.
\end{abstract}

\section{Introduction}

Pseudo-fractional auto-regressive moving average (ARMA) modelling is a pole-zero modelling of fractional linear systems. These are described by fractional differential equations in the continuous-time case or auto-regressive integrated moving average (ARIMA) models in the discrete-time case. The first case is based on the definition of fractional differintegration, whereas the second deals with the fractional differencing that is a fractional version of the well-known finite differences. These systems are characterised by having a long memory that cannot be explained by the usual linear systems that have short memory (exponential). The desire of finding a theoretical base for such systems led to the fractional calculus that has recently received a great deal of attention in the scientific literature, through the publication of books, special issues of journals, review articles, as well as a very large number of research papers. The interest in fractional calculus comes from the fact that it provides foundations for the understanding of several natural phenomena and the basic theory for building models for the systems underlying them. However, adoption of the fractional calculus by the physicists and engineering community was inhibited historically by the lack of clear experimental evidence for its need and by the difficulty in constructing simple models for simulation or even implementation of simple fractional systems. Fractional calculus is almost as old as the common calculus, but only since 30 years ago it has been a subject of specialised publications and conferences.

The basic building block of this kind of systems is the non-integer order derivative and integral that have been

\footnotetext{
(C) The Institution of Engineering and Technology 2006 doi:10.1049/iet-cta:20050001

Paper first received 5th January 2005 and in revised form 15th February 2006 M.D. Ortigueira is with UNINOVA and DEE, Faculdade de Ciências e Tecnologia da Universidade Nova de Lisboa, Campus da FCT da UNL, Quinta da Torre, Caparica 2829-516, Portugal and also with L2F - INESC ID, Rua Alves Redol, 9, 2, Lisboa 1000-029, Portugal

A.J. Serralheiro is with L2F - INESC ID, Rua Alves Redol, 9, $2^{\circ}$, Lisboa 1000029, Portugal and also with the Academia Militar, Rua Gomes Freire, Lisboa 1150-175, Portugal

E-mail: antonio.serralheiro@inesc-id.pt
}

approximated by fractional powers of the backward difference or the bilinear transformations - the former is exactly the building block of the fractional differencing, as said earlier. However, these approximations are described infinite impulse response (IIR) systems with nonrational transfer functions. For these, ARMA models are only approximations. However, the usefulness of ARMA models makes them very interesting when constructing discrete-time approximating models for fractional systems. In the last few years, a lot of attempts to obtain such models have been done [1-5]. However, it is not clear how to perform such modelling and how to choose the most suitable orders, although there are a lot of algorithms, mainly in the stochastic case. In impulse response modelling, the well-known Padé algorithm is frequently used [6]. In this paper, we shall not be concerned with the estimation task involved in ARMA modelling; instead, we are going to look at the underlying structure of the ARMA model in order to find alternative relations to obtain the ARMA parameters from the impulse response. To be more specific, consider the usual theoretical approach for computing the ARMA $(N, M)$ parameters from the impulse response, $h_{n}$

$$
\sum_{i=0}^{N} a_{i} h_{j-i}= \begin{cases}\mathrm{b}_{\mathrm{j}} & j \geq 0, \ldots, M \\ 0 & j>M\end{cases}
$$

where $a_{i}$ and $b_{i}$ are, respectively, the AR and MA parameters. A close look into (1) shows that, to compute the ARMA $(N, M)$ parameters, we only need the first $N+M+1$ values of the impulse response $\{h(n)$, $n=0, \ldots, N+M$. Here, we propose a new description of the double Levinson recursion presented in the work of Ortigueira and Tribolet [7]. The algorithm consists of the recursive solution of the system obtained from (1) with $j=M$ to $j=N+M$ for the AR coefficients followed by the use of the first $M+1$ equations to obtain the MA parameters. This algorithm gives the possibility of determining the orders of the systems when looking at the pattern formed by a sequence of coefficients. We applied this algorithm in (pseudo) fractional ARMA modelling and observed that the pattern does not clearly point the orders, but give some insights into minimum orders. 


\section{Algorithm}

The procedure we are going to describe is very similar to the one presented by Ortigueira and Tribolet [7] but, instead of using the matrix formulation, we adopt a Schur-like description [8], as it is more direct and easier to implement. To begin with, we consider (1) and introduce a function $f_{M}^{N}(j), j=0,1, \ldots$, given by

$$
f_{M}^{N}(j)=\sum_{i=0}^{N} a_{M}^{N}(i) h_{j+M-i}
$$

where we enhance the orders $N$ and $M$. According to (1) this function has gaps for $j=1,2, \ldots, N$. For $N=0$, we thus have

$$
f_{M}^{0}(j)=h_{j+M} \quad j=0,1, \ldots
$$

The algorithm described by Ortigueira and Tribolet [7] uses an adjoint system [9]. Here, we introduce an adjoint function defined by

$$
g_{M}^{N}(j)=\sum_{i=0}^{N} \gamma_{M}^{N}(i) h_{N-j+M-i}
$$

with

$$
g_{M}^{0}(j)=h_{-j+M} \quad j=0,1, \ldots
$$

As it is clear, $g_{M}^{N}(j)$ has gaps for $j=1,2, \ldots, \mathrm{N}$, too. The solution of (1) is recursively constructed for successive values of $N$ from $N=1$ to $N=N_{0}$, where $N_{0}$ is a positive integer. To do this, assume that we have constructed the $(N-1)$ th order functions $f_{M}^{N-1}(j)$ and $g_{M}^{N-1}(j) j=0$, $1, \ldots$ We will construct the $N$ th order functions by the recursions

$$
f_{M}^{N}(j)=f_{M}^{N-1}(j)+K_{M}^{N} g_{M}^{N-1}(N-j)
$$

and

$$
g_{M}^{N}(j)=g_{M}^{N-1}(j)+H_{M}^{N} f_{M}^{N-1}(N-j)
$$

where $K_{M}^{N}$ and $H_{M}^{N}$ are obtained by forcing both functions to have a gap at $j=N$. We obtain

$$
\begin{aligned}
& K_{M}^{N}=-\frac{f_{M}^{N-1}(N)}{g_{M}^{N-1}(0)} \\
& H_{M}^{N}=-\frac{g_{M}^{N-1}(N)}{f_{M}^{N-1}(0)}
\end{aligned}
$$

As it is easy to verify, we have also

$$
g_{M}^{N}(0)=f_{M}^{N-1}(0)\left(1-K_{M}^{N} \cdot H_{M}^{N}\right)
$$

If the system with impulse response $h_{n}$ is really an ARMA $\left(N_{0}, M_{0}\right)$, we will have

$$
b_{j}=f_{M_{0}}^{N_{0}}\left(j-M_{0}\right) \quad j=0, \ldots, M_{0}
$$

For the AR coefficients we use (2), (4), (6) and (7) and the $K_{M}^{N}$ and $H_{M}^{N}$ sequences to obtain the so-called double Levinson recursion [7]

$$
a_{M}^{N}(i)=a_{M}^{N-1}(i)+K_{M}^{N} \gamma_{M}^{N-1}(N-i)
$$

and

$$
\gamma_{M}^{N}(i)=\gamma_{M}^{N-1}(i)+H_{M}^{N} a_{M}^{N-1}(N-i)
$$

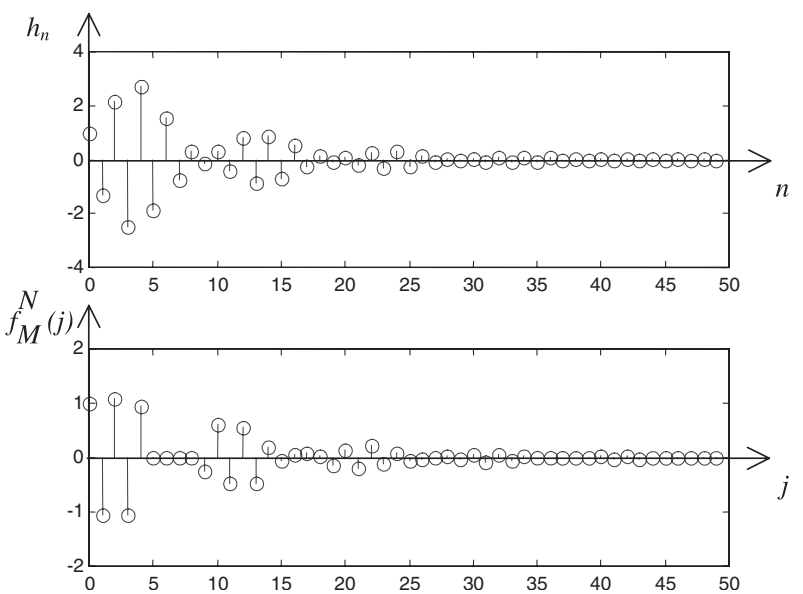

Fig. 1 Impulse response $h_{n}$ of a $\operatorname{ARMA}(6,4)$ system (top) and $f_{M}^{N}(j), j=0,1, \ldots$ (bottom) of an $\operatorname{ARMA}(4,4)$ model

with $i=0,1, \ldots, N . K_{M}^{N}$ and $H_{M}^{N}$ are generalised reflection coefficients. To get some insights into the algorithm, we will describe an application to an $\operatorname{ARMA}(6,4)$ model. This has the impulse response represented in the upper half of Fig. 1. In the lower part, we present the function $f_{M}^{N}(j), j=0,1, \ldots$ for $N=M=4$. As it can be seen, we inserted 4 gaps, but there are still non-zero values for $j>4$, clearly meaning that we are using too low orders.

In Fig. 2, we repeat the situation, but now the constructed model is an $\operatorname{ARMA}(6,4)$. As it can be seen, now the gaps collapsed the function for all the values above $M$. The non-zero values are the MA coefficients.

The double Levinson recursion supplies us with a very important result, which will be useful in determining the orders of the model. This result is stated in the following theorem (for proof refer to Machado [2]).

Theorem: Let $M \geq 0$ be an integer constant and $A_{M}^{N}(z)$ and $\Gamma_{M+1}^{N}(z)$ the $Z$ transforms of $a_{M}^{N}(i)$ and $\gamma_{M+1}^{N}(i)$. The $N$ th degree polynomials $A_{M}^{N}(z)$ and $\Gamma_{M+1}^{N}(z)$ corresponding to $M$ and $M+1$ zeros, respectively, are, up to a constant, reverse polynomials

$$
\Gamma_{M+1}^{N}(z)=\phi \cdot z^{-N} \cdot A_{M}^{N}\left(z^{-1}\right)
$$

$\phi$ being the last coefficient of $\Gamma_{M+1}^{N}(z)$.
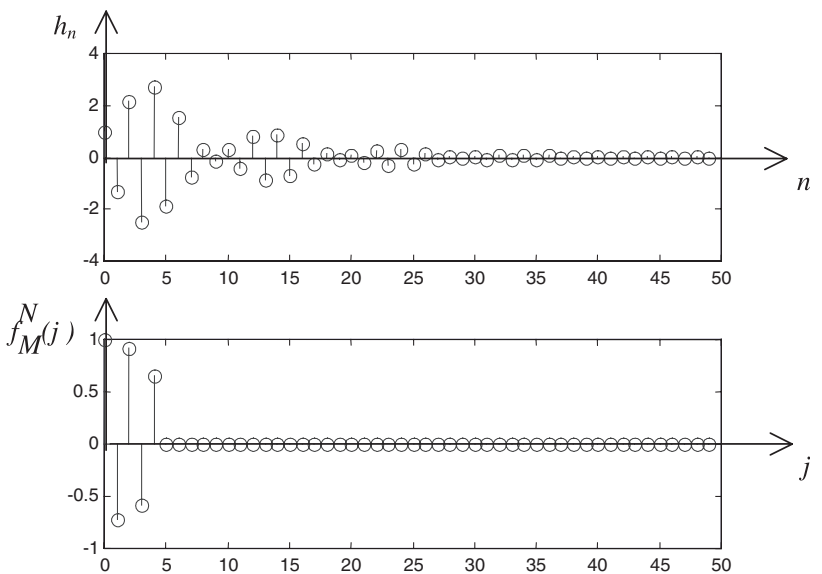

Fig. 2 Impulse response $h_{n}$ of an $A R M A(6,4)$ system (top) and $f_{M}^{N}(j), j=0,1, \ldots$ (bottom) of an ARMA(6,4) model 
Table 1: $H_{M}^{N} \cdot K_{M}^{N}$ product pattern for the AR(3) case

\begin{tabular}{llllc}
\hline$N$ & $M$ & & & \\
& 1 & 2 & 3 & 4 \\
\hline 1 & -0.012 & $h v$ & 0.0082 & -2.5803 \\
2 & 0.6571 & $h v$ & 1.1446 & 1.0401 \\
3 & 1 & $h v$ & 1 & 1 \\
4 & $h v$ & 6.6935 & 0.2955 & -2.1107 \\
\hline
\end{tabular}

As consequence of this theorem, we obtain

$$
H_{M+1}^{N} \cdot K_{M}^{N}=1
$$

Its proof being immediate from the theorem. This result is very interesting as it allows us to compute the correct orders:

- it is enough to run the algorithm for $N, M$ values ranging from 0 to $N_{0}, M_{0}$ higher than the expected orders;

- for the correct AR order and the correct plus one MA order the product is one.

To exemplify these assertions, we will consider the following two examples.

Example 1: AR case: Consider an AR(3) with coefficients $a=\left[\begin{array}{lll}1-1.0871 & 1.1961-0.4512\end{array}\right]$. In Table 1 , we show the $H_{M}^{N} \cdot K_{M}^{N}$ product pattern for $N, M=0,1,2,3,4$, where 'hv' means a high value obtained when the determinant of the underlying matrix is close to zero (small values of $f_{M}^{N}(0)$ after a product almost equal to one). This happens when the orders are oversized. From the table, we conclude easily that the system is indeed an $\operatorname{AR}(3)$. It is interesting to remark that if we compute the poles and zero corresponding to an $\operatorname{ARMA}(4,1)$, the extra pole is cancelled by the zero (as it should be).

Example 2: ARMA case: We consider now an $\operatorname{ARMA}(3,2)$ system defined by the previous AR parameters (Example 1) and with $b=\left[\begin{array}{lll}1 & 1.2 & -1.6\end{array}\right]$ as MA parameters. As before, Table 2 showing the $H_{M}^{N} \cdot K_{M}^{N}$ product pattern for $N$, $M=0,1,2,3,4$, suggests the correct orders. It is important to refer that:

(a) although the system is not minimum phase, we obtain the correct MA parameters;

(b) if the MA order is the correct one but the AR one is oversized, there is no problem as the extra coefficients are zero.

The application of this algorithm to the pole/zero modelling of integer order continuous-time systems is also possible. To do it, we only have to substitute above the impulse response by the sequence $h_{n}=(-1)^{n} / n ! m_{n}$,

Table 2: $\quad H_{M}^{N} \cdot K_{M}^{N}$ product pattern for the ARMA $(3,2)$ case

\begin{tabular}{lllll}
\hline$N$ & \multicolumn{1}{l}{$M$} & & & \\
& 1 & 2 & 3 & \multicolumn{1}{l}{4} \\
\hline 1 & -0.0592 & 62.5179 & 0.0652 & -1.7805 \\
2 & 0.1984 & 0.9592 & 0.8598 & 0.9575 \\
3 & 0.0422 & -0.9930 & 1 & 1 \\
4 & 0.0165 & 0 & hv & 0.8159 \\
\hline
\end{tabular}

$n=0,1, \ldots$, where $m_{n}$ is the sequence of the momenta of the impulse response of the continuous-time system given by $m_{n}=\int_{0}^{\infty} h(t) t^{\mathrm{n}} \mathrm{d} t$.

The importance of (14) lies on the bridge it establishes between two different MA order polynomials. As a consequence of this theorem we have, from (13) [10]

$$
A_{M}^{N-1}(z)=A_{M-1}^{N}(z)+\mu_{M}^{N} z^{-1} \cdot A_{M-1}^{N-1}(z)
$$

where $\mu_{M}^{N}$ is obtained by forcing the $N$ th order coefficient to be zero

$$
\mu_{M}^{N}=-\frac{a_{M-1}^{N}(N)}{a_{M-1}^{N-1}(N-1)}
$$

The recursion (16) is very interesting as it allows us to compute the AR part of a given $\operatorname{ARMA}\left(N_{0}, M_{0}\right)$ from a sequence of $\mathrm{AR}$ models with orders ranging from 1 to $N_{0}+M_{0}$. Using (2), we obtain

$$
f_{M}^{N-1}(j)=f_{M-1}^{N}(j+1)+\mu_{M}^{N} f_{M-1}^{N-1}(j)
$$

that allows us to compute the MA parameters from $f_{0}^{N}(j)$, resulting from the Levinson recursion (12) with $M=0$. In our applications, we preferred to compute the MA parameters from (1), though.

We applied the recursion (16) to the model used in Example 1. Immediately at the first recursion $(M=1)$, all the polynomial with degree greater than or equal to 4 reproduced the AR polynomial we were looking for. All the extra coefficients were zero. The same happened with the second at $M=2$. When we go beyond the correct MA order, the coefficient (17) becomes very high because of a division by a very small value (theoretically, zero).

\section{Application to pseudo-fractional modelling}

In this section, we assess the estimation of ARMA models for approximating discrete-time fractional models. We considered the fractional difference

$$
H_{\mathrm{bd}}(z)=\left(1-z^{-1}\right)^{\alpha}, \quad|z|>1
$$

and the Tustin (bilinear)

$$
H_{\mathrm{bil}}(z)=\left(\frac{1-z^{-1}}{1+z^{-1}}\right)^{\alpha}|z|>1
$$
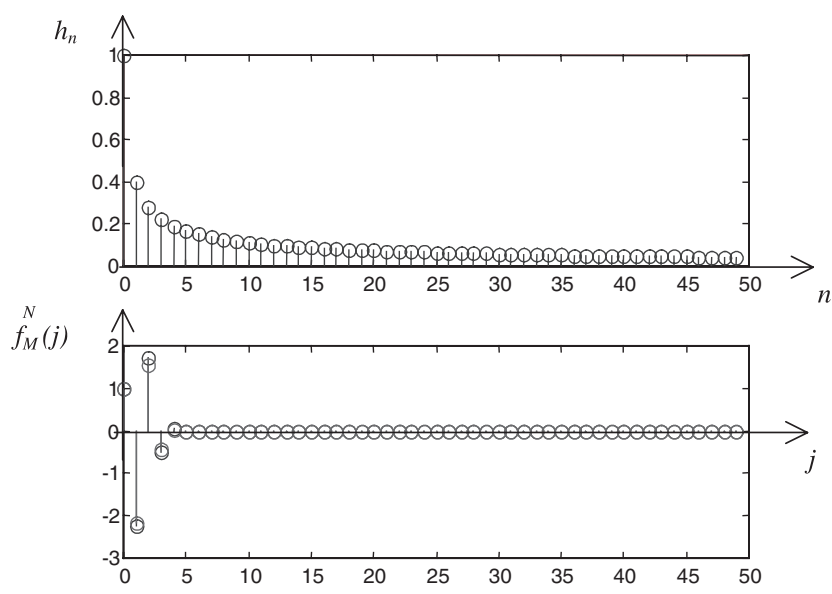

Fig. 3 Impulse response $h_{n}$ of a backward difference system (top) and $f_{M}^{N}(j), j=0,1, \ldots$ (bottom) of an $\operatorname{ARMA}(4,4)$ and an ARMA $(6,4)$ models 

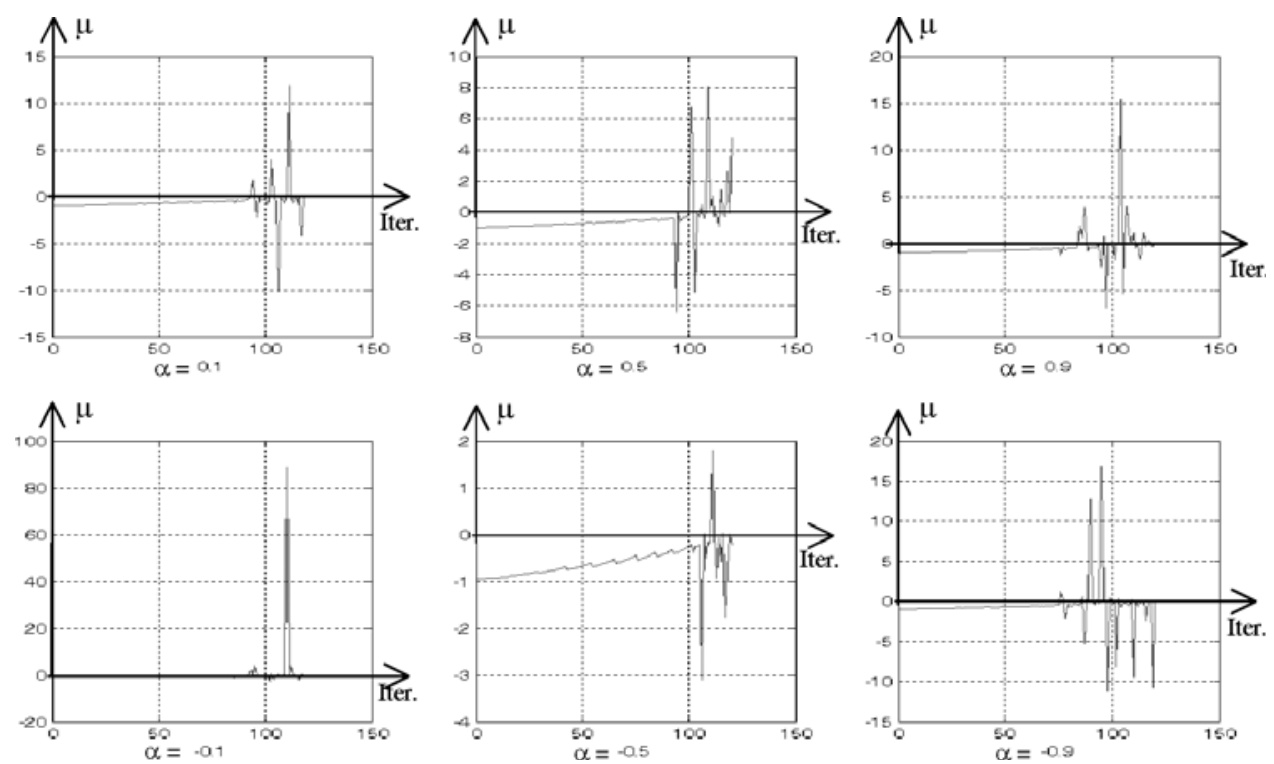

Fig. 4 Behaviour of the $\mu_{M}^{N}$ as a tool for orders choice, for the differentiator (above) and integrator (below), with $\alpha=0.1$ (left), $\alpha=0.5$ (middle) and $\alpha=0.9$ (right)

'Iter' means model iteration

as approximations to the $\alpha$-order differintegrator. For each one, we applied the algorithms described earlier and tried to find patterns that pointed us towards minima orders. We used only values of $|\alpha|<1$, because the other cases correspond to join integer order poles and/or zeros to the ARMA model obtained with the fractional part of $\alpha$.

The introduction of gaps in the function $f_{M}^{N}(j)$, $j=0,1, \ldots$ is presented in Fig. 3 for backward differences with order $\alpha=-0.4$. We present the results obtained with an $\operatorname{ARMA}(6,4)$ and $\operatorname{ARMA}(4,6)$. As it can be seen, the values of the function above four are almost zero, meaning that, although the original system is not ARMA, it can be modelled with an ARMA. For the bilinear case, the results are similar. With this in mind, we are going to study the behaviour of product $H_{M+1}^{N} \cdot K_{M}^{N}$ through the recursion progression.

The product $H_{M+1}^{N} \cdot K_{M}^{N}$ pattern does not tell much but, and as in the previous tables, we find that the lower diagonal values were smaller than those in the upper diagonal in both
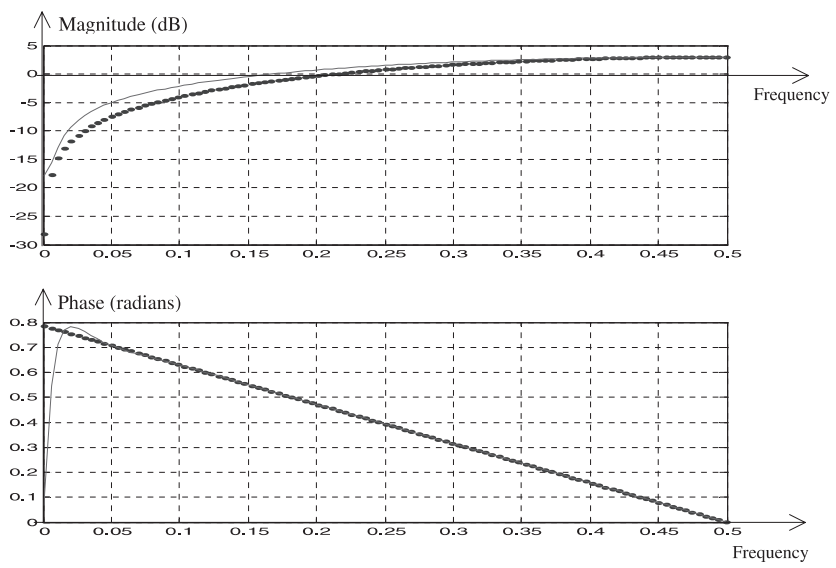

Fig. $5 \operatorname{ARMA}(9,9)$ frequency response plots (solid lines) for the backward difference, with $\alpha=0.5$

Upper graph is the magnitude response (in $\mathrm{dB}$ ) and the lower graph is the phase diagram (in radian); dotted line curves correspond to the exact model cases (19) and (20). This suggests that the MA order is more important than the AR one. In Tables 3 and 4 , to better illustrate the behaviour of such patterns for the backwards difference and bilinear, all the values less than 0.9 were represented by a ' 0 ', whereas values between 0.9 and 1.1 were represented by ' 1 ' and, finally, the values above 1.1 were represented by ' 2 ' (Figs. 4-6).

To try to find other insights into the orders, we ran the recursive algorithm, allowing us to conclude that:

(a) small values of the coefficient $\mu_{M}^{N}$ in (17) point to correct $N$ and $M$ orders (Fig. 4);

(b) very high values of $\mu_{M}^{N}$ mean that, at least the MA order is higher than the correct. In this situation we must decrease it. This situation corresponds to unstable model;

(c) the observation of the $\mu_{M}^{N}$ pattern suggests an $\operatorname{ARMA}(N$, $N)$ model;

(d) in the difference case, the poles and zeros of the $\operatorname{ARMA}(N, N)$ model are always positive and interlaced;
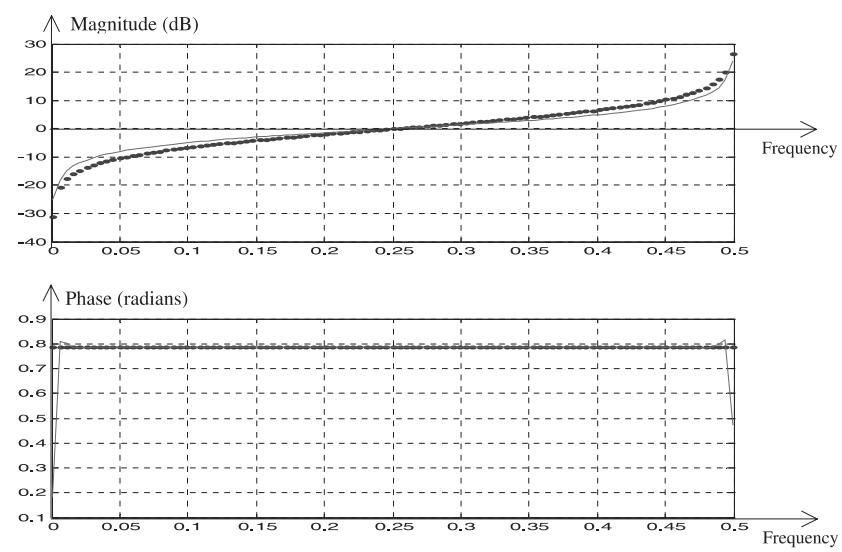

Fig. $6 \operatorname{ARMA}(9,9)$ frequency response plots (solid lines) for bilinear, with $\alpha=0.5$

Upper graph is the magnitude response (in $\mathrm{dB}$ ) and the lower graph is the phase diagram (in radian); dotted curves correspond to the exact model 
Table 3: $\quad H_{M}^{N} \cdot K_{M}^{N}$ product pattern for the ARMA $(10,10)$ corresponding to the backward difference case, where zeros (0) represent values less than 0.9 , ones (1) represent values between 0.9 and 1.1 and twos (2) represent values greater than $\mathbf{1 . 1}$

\begin{tabular}{ccccccccccc}
\hline$N$ & $M$ & & & & & & & & & \\
& 1 & 2 & 3 & 4 & 5 & 6 & 7 & 8 & 9 & 10 \\
\hline 1 & 0 & 2 & 2 & 2 & 1 & 1 & 1 & 1 & 1 & 1 \\
2 & 0 & 0 & 2 & 2 & 2 & 2 & 2 & 1 & 1 & 1 \\
3 & 0 & 0 & 0 & 2 & 2 & 2 & 2 & 2 & 2 & 2 \\
4 & 0 & 0 & 0 & 0 & 2 & 2 & 2 & 2 & 2 & 2 \\
5 & 0 & 0 & 0 & 0 & 0 & 2 & 2 & 2 & 2 & 2 \\
6 & 0 & 0 & 0 & 0 & 0 & 0 & 2 & 2 & 2 & 2 \\
7 & 0 & 0 & 0 & 0 & 0 & 0 & 0 & 2 & 2 & 2 \\
8 & 0 & 0 & 0 & 0 & 0 & 0 & 0 & 0 & 2 & 2 \\
9 & 0 & 0 & 0 & 0 & 0 & 0 & 0 & 0 & 0 & 2 \\
10 & 0 & 0 & 0 & 0 & 0 & 0 & 0 & 0 & 0 & 0 \\
\hline
\end{tabular}

(e) in the bilinear case, the poles and zeros of the $\operatorname{ARMA}(N, N)$ model are symmetric;

(f) our simulations pointed out to values of $N$ from 7 to 10 . For most situations, the approximation is good in both time and frequency domains.

(g) smaller values of $\alpha$ results in better amplitude frequency response approximations to the exact model, the largest amplitude deviations occurring in the low-frequency regions for the difference case (Figs. 5 and 6). For the cases where $|\alpha|>0.5$, leading to a worst amplitude response modelling, one can always model them as a combination of two models, thus forcing the 'new' fractional to be of order $(1-\alpha)$. The additional integer term corresponds to either an integrator or a differenciator that results in an extra $\left(1-z^{-1}\right)^{-1}$ or $\left(1-z^{-1}\right)$ factor, respectively.

We made also a study of the pole-zero distribution (Figs. 7 and 8) and found that:

(a) in the backward difference case (models $N, N$ ) the poles and zeros are positive real. With higher-orders complex poles and zeros may appear (above $N=M=9$ );

Table 4: $\quad H_{M}^{N} \cdot K_{M}^{N}$ product pattern for the ARMA $(10,10)$ corresponding to the bilinear case, where zeros $(0)$ represent values less than 0.9 , ones (1) represent values between 0.9 and 1.1 and twos (2) represent values greater than 1.1

\begin{tabular}{|c|c|c|c|c|c|c|c|c|c|c|}
\hline \multirow[t]{2}{*}{$N$} & \multicolumn{10}{|c|}{$M$} \\
\hline & 1 & 2 & 3 & 4 & 5 & 6 & 7 & 8 & 9 & 10 \\
\hline 1 & 0 & 2 & 0 & 2 & 0 & 2 & 0 & 2 & 0 & 2 \\
\hline 2 & 0 & 0 & 2 & 0 & 2 & 0 & 2 & 0 & 2 & 1 \\
\hline 3 & 0 & 0 & 0 & 2 & 0 & 2 & 0 & 2 & 0 & 2 \\
\hline 4 & 0 & 0 & 0 & 0 & 2 & 0 & 2 & 0 & 2 & 0 \\
\hline 5 & 0 & 0 & 0 & 0 & 0 & 2 & 0 & 2 & 0 & 2 \\
\hline 6 & 0 & 0 & 0 & 0 & 0 & 0 & 2 & 0 & 2 & 0 \\
\hline 7 & 0 & 0 & 0 & 0 & 0 & 0 & 0 & 2 & 0 & 2 \\
\hline 8 & 0 & 0 & 0 & 0 & 0 & 0 & 0 & 0 & 2 & 0 \\
\hline 9 & 0 & 0 & 0 & 0 & 0 & 0 & 0 & 0 & 0 & 2 \\
\hline 10 & 0 & 0 & 0 & 0 & 0 & 0 & 0 & 0 & 0 & 0 \\
\hline
\end{tabular}

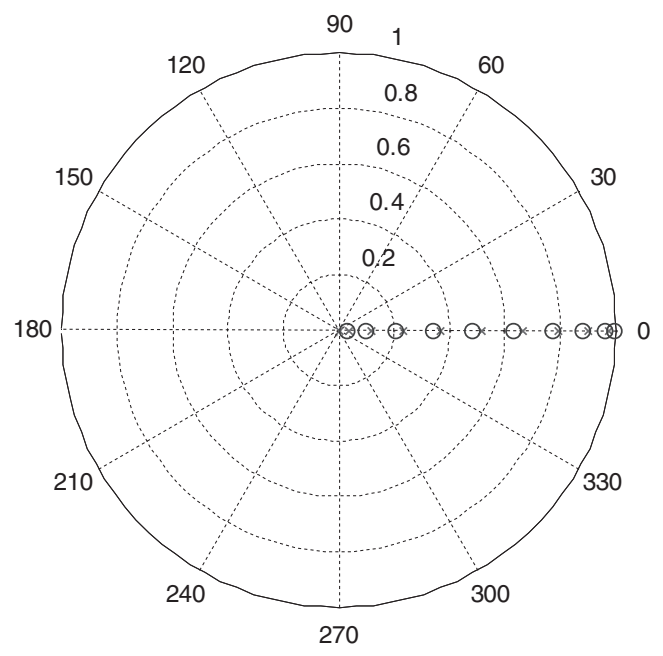

Fig. $7 \operatorname{ARMA}(N, M)$ polar pole-zero plots for the backward differences with $\alpha=0.8$ for $N, M=10$

(b) in the bilinear case, the poles and zeros are real. We did not find complex poles or zeros, unless $N=$ $M>16$;

(c) in the bilinear case, and for some $\alpha$, we obtained unstable models for some $N, M<N$;

(d) if the pole order is the same of the zero order, the poles and zeros are interlaced. In the bilinear case, they are symmetric to each other;

(e) if the orders are not equal, the 'extra' poles or zeros tend to appear near zero.

Although the last statement is more or less natural, statement (d) deserves some considerations. A presence of a pole $(\alpha<0)$ or a zero $(\alpha>0)$ near $z=1$ is needed, as it is the branch point of the transfer function and it has a very large influence on the function. The interlaced pole/zero pairs is needed because we know that zeros, inside the unit circle, decrease the amplitude and increase the phase, whereas poles increase the amplitude and decrease the phase. So the doublets pole/zero determine the variation of the amplitude and phase. We may ask why they are over the real axis. If they were not at the real axis, they would contribute to the appearing of peaks and valleys, because the amplitude and phase changed faster and the doublet effect was not so clear.

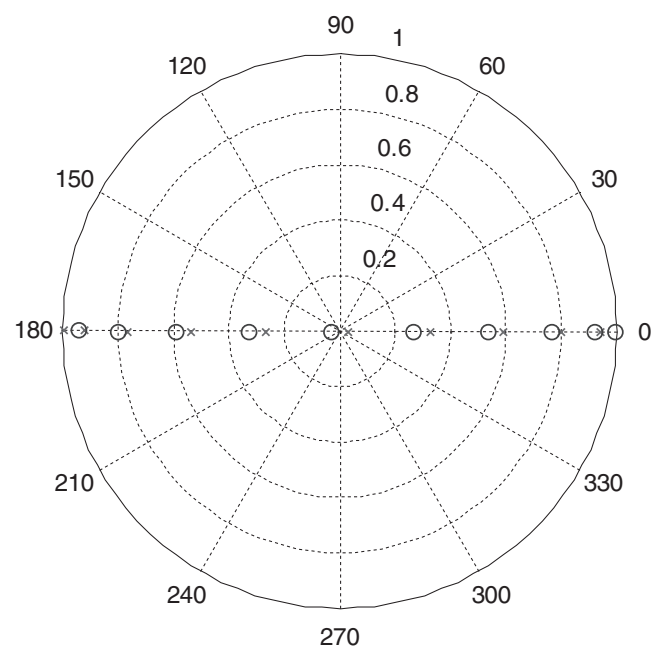

Fig. $8 \operatorname{ARMA}(N, M)$ polar pole-zero plots for the bilinear with $\alpha=0.8$ for $N, M=10$ 


\section{Conclusions}

The algorithm we have just presented gives a simple way of computing the AR and MA parameters of an ARMA model from a given impulse response, of a not necessarily ARMA, linear system. The algorithm is recursive and gives insights into the orders computation. We applied it to model two special cases of fractional linear systems: the systems with transfer functions that are fractional powers of the backward difference and bilinear transformations. We presented some examples. From them, we may conclude that we cannot give widely valid prescriptions on the modelling orders: different fractional orders imply different ARMA models. However, it seems that the pole-zero pair (doublet) has an important job in the modelling performance.

\section{References}

1 Vinagre, B.M., Podlubny, I., Hernandez, A., and Feliu, V.: 'Some approximations of fractional order operators used in control theory and applications', Fract. Calc. Appl. Anal., 2000, 3, (3), pp. 231-248
2 Machado, J.A.T.: 'Discrete-time fractional-order controllers', Fract. Calc. Appl. Anal. J., 2001, 1, pp. 47-66

3 Chen, Y.Q., and Vinagre, B.M.: 'A new IIR-type digital fractional order differentiator', Signal Process., 2003, 83, pp. 2359-2365

4 Barbosa, R.S., Machado, J.A.T., and Ferreira, I.M.: 'Least-squares design of digital fractional-order operators'. FDA '2004 1st IFAC Workshop on Fractional Differentiation and Its Applications, Bordeaux, France, July 19-21, 2004

5 Ostalczyk, P.: 'Fundamental properties of the fractional-order discrete-time integrator', Signal Process., 2003, 83, pp. 2367-2376

6 Kumar, K.: 'On the identification of autoregressive moving average models', J. Control Intell. Syst., 2000, 28, (2), pp. 41-46

7 Ortigueira, M.D., and Tribolet, J.M.: 'On the double Levinson recursion formulation of ARMA spectral estimation'. Proc. IEEE ICASSP, 1983

8 Robinson, E.A., and Treitel, S.: 'Maximum entropy and the relationship of the partial autocorrelation to the reflection coefficients of a layered system', IEEE Trans. ASSP, 1980, 28, (2), pp. 224-235

9 Carayannis, G., Kaloupsidis, N., and Manolakis, D.G.: 'Fast algorithms for a class of linear equations', IEEE Trans. ASSP, 1982 30, (2), pp. 227-239

10 Ortigueira, M.D.: 'ARMA realization from the reflection coefficient sequence', Signal Process., 1993, 32, (3), pp. 329-342 